\title{
Desenvolvimento larval inicial de Helostoma temminckii Cuvier \& Valenciennes (Helostomatidae, Perciformes)
}

\author{
Wilson Treger Zydowicz de Sousa ${ }^{1}$ \\ William Severi ${ }^{1}$
}

\begin{abstract}
Initial larval development of Helostoma temminckii Cuvier \& Valenciennes (Helostomatidae, Perciformes). This work presents the characterization of larval external morphology of the kissing gourami Helostoma temminckii, an aquarium fish of the family Helostomatidae, reared all over the world. A total of 38 larvae (standard length between 3.2 and $10.1 \mathrm{~mm}$ ), obtained through natural reproduction carried out in the Fish Culture Station of the Federal Rural University of Pernambuco, were used. The morphometric measurements pre-dorsal, pre-pectoral and pre-anal distances, and body height varied positively with standard length, and body proportions varied between 30.8 and $51.2 \% ; 12.9$ and $37.9 \% ; 38.2$ and $58.3 \%$, and 17.2 and $30.43 \% \mathrm{CP}$, respectively. The eye diameter varied positively with the length of the head (CC), but its body proportion decreased with growth, varying between 63.3 and $30.2 \% \mathrm{CC}$, within fish lenghts from 3.2 to $10.1 \mathrm{~mm}$, respectively. The external morphological characterization of larvae with 3.9, 6.6, 7.7, 8.0 and $10.1 \mathrm{~mm} \mathrm{CP}$ are presented, showing the notochord flexion (CP between 5.5 and $6.8 \mathrm{~mm}$ ), the appearance of rays and formation of the caudal (CP between 5.5 and $7.5 \mathrm{~mm}$ ), dorsal (CP above $7.5 \mathrm{~mm}$ ), anal (larger than $7.5 \mathrm{~mm} \mathrm{CP}$ ) and pectoral fins (larger than $8 \mathrm{~mm} \mathrm{CP}$ ); the modification of body shape and pigmentation absence. Larvae with $10.1 \mathrm{~mm}$ CP don't show completely developed dorsal and anal fins, with their definitive number of rays. The opening of mouth and anus, and reduction of the yolk-sac were observed in larvae with CP between 6.5 and $7.5 \mathrm{~mm}$. Such information is important for larval rearing of the species, besides providing data for comparative ontogenic analysis of other Perciformes.

KEY WORDS. Helostoma temminckii, kissing gourami, larval development
\end{abstract}

O beijador Helostoma temminckii Cuvier e Valenciennes, 1831 é um peixe de água doce da familia Helostomatidae, largamente cultivado como espécie ornamental por aquariofilistas de todo o mundo. Tem, como características mais evidentes, uma coloração róseo-prateada, sem pigmentação no corpo ou nadadeiras, e o hábito de "beijar" os companheiros.

A espécie é a única do gênero Helostoma reconhecida por NELSON (1994), com distribuição endêmica ao sudeste asiático. É um filtrador especializado, com rastros branquiais finos e lábios dotados de dentes ósseos, empregados na raspagem de algas de substratos.

Sua reprodução em aquário ocorre naturalmente, sob condições nutricionais adequadas, mediante fecundação externa, sem construção de ninhos de bolhas, como em outros anabantoídeos. A fecundidade em fêmeas plenamente maduras pode ultrapassar 300 óvulos, com a eclosão de larvas ocorrendo dentro de 24 horas, sob temperaturas acima de $25^{\circ} \mathrm{C}$ (AXELROD \& SCHULTZ 1990).

1) Laboratório de Ictiologia, Departamento de Pesca, Universidade Federal Rural de Pernambuco. Avenida Dom Manuel de Medeiros 68, Dois Irmãos, 52171-900 Recife, Pernambuco, Brasil. E-mail: wseveri@truenet.com.br 
A identificação de ovos e larvas de peixes, aliada à caracterização de aspectos morfológicos e fisiológicos dos mesmos e suas alterações ao longo do desenvolvimento, fornecem informações relevantes acerca da biologia e ecologia das espécies, constituindo conhecimento imprescindivel ao estudo da biologia pesqueira e do cultivo das mesmas (BLAXTER 1984; LASKER 1987; HoUdE 1987).

O conhecimento sobre o desenvolvimento larval de peixes de água doce é limitado. Apesar do grande número de espécies ornamentais de elevado valor comercial dentre os Cypriniformes, Characiformes e Siluriformes, largamente reproduzidos e cultivados em aquários, pouco desse conhecimento tem sido utilizado em estudos de ecologia e desenvolvimento de ovos e larvas (RICHARDS 1985). Mesmo entre grupos de distribuição mais ampla, como os Perciformes, menos de 4\% das espécies têm suas larvas conhecidas, e dados sobre espécies da subordem Anabantoidei não constam da revisão de MOSER et al. (1984), sendo escassos na literatura.

Este trabalho tem como objetivo caracterizar o desenvolvimento inicial de larvas de $H$. temminckii, através da descrição de sua morfologia externa e morfometria.

\section{MATERIAL E MÉTODOS}

Larvas de H. temminckii $(\mathrm{n}=38)$ de diferentes tamanhos, com comprimento padrão (CP) entre 3,2 e $10,1 \mathrm{~mm}$, e idades variando entre 2,5 horas e 15 dias pós-eclosão, foram obtidas através de reprodução natural em tanques de 200 litros, realizada na Base de Piscicultura da Universidade Federal Rural de Pernambuco.

A caracterização de seu desenvolvimento inicial foi realizada com base numa série ontogênica (AHLSTROM \& MOSER 1976) composta de indivíduos em diferentes estágios ontogênicos, separados conforme o grau de flexão da notocorda, nas fases de pré-flexão, flexão e pós-flexão (KENDALL et al. 1984).

Os indivíduos foram caracterizados morfometricamente de acordo com as seguintes medidas: comprimento padrão $(\mathrm{CP})$, altura do corpo (AC), comprimento da cabeça (CC), distância pré-dorsal (DPD), distância pré-peitoral (DPP), distância pré-anal (DPA) e diâmetro do olho (DO). As medidas AC, CC, DPD, DPP e DPA foram relacionadas com $\mathrm{CP}$ e $\mathrm{DO}$ com $\mathrm{CC}$, através da equação $\mathrm{Y}=a \mathrm{X}+b$.

A variação de suas proporções ao longo do desenvolvimento foi analisada em relação ao comprimento padrão, exceto para o diâmetro do olho, expresso em relação ao comprimento da cabeça, classificando-se as fases segundo as categorias propostas por LEIS \& TRNSKI (1989).

\section{RESULTADOS}

\section{Caracterização morfológica das larvas}

As larvas de Helostoma temminckii, ao eclodir, medem entre 3,0 e 3,5 mm CP.

A larva com 3,0 mm CP (Fig. 1A) encontra-se no estágio de pré-flexão, com notocorda retilínea. Uma membrana embrionária (finfold) envolve toda a extensão dorsal e ventral, sem diferenciação entre as nadadeiras dorsal, caudal e anal ou de seus raios. A cabeça apresenta forma arredondada, com olho grande e densamente 


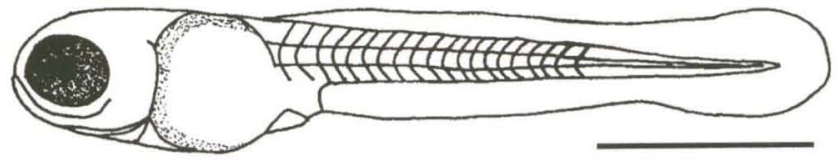

A

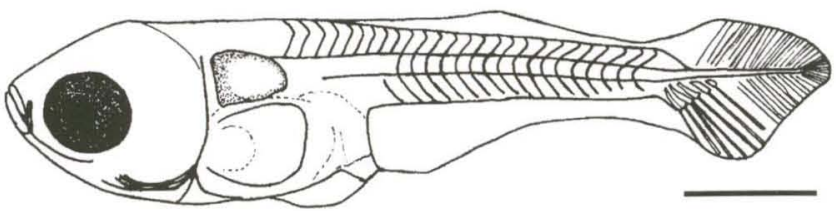

B

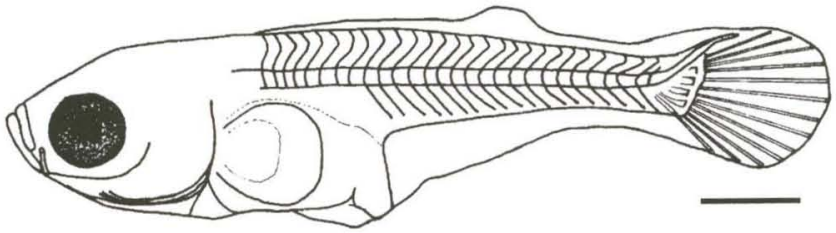

C

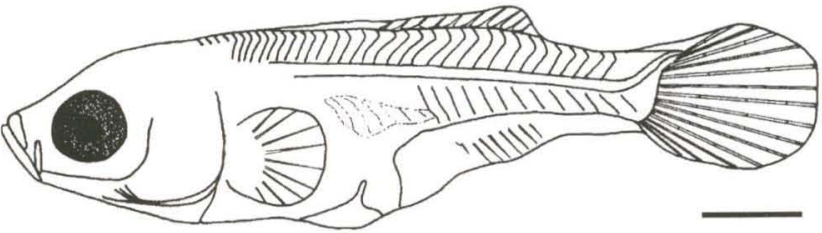

D

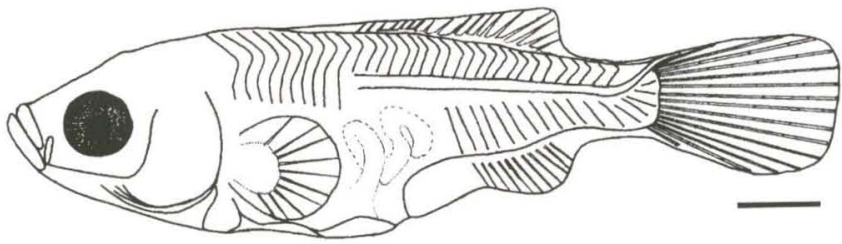

$\mathbf{E}$

Fig. 1. Larvas de Helostoma temminckii: (A) $3,9 \mathrm{~mm} \mathrm{CP}$ (pré-flexão); (B) $6,6 \mathrm{~mm} \mathrm{CP}$ (flexão); (C) 7,7 mm CP (pós-flexão); (D) 8,0 mm CP (pós-flexão); (E) 10,1 mm CP (pós-flexão). O traço corresponde a $1,0 \mathrm{~mm}$.

pigmentado. $\mathrm{O}$ corpo não apresenta pigmentação, com reserva vitelínica ocupando toda a cavidade abdominal, sem diferenciação do trato digestivo. A segmentação dos miômeros é evidenciada por transparência.

As larvas medindo entre 5,0 e 6,7 mm CP (Fig. 1B) encontram-se no estágio de flexão. A cabeça apresenta-se mais pontiaguda, com boca e opérculo bem desenvolvidos e reserva vitelínica em absorção. A membrana embrionária encon- 
tra-se diferenciada na nadadeira dorsal e os raios da nadadeira caudal estão em formação. A nadadeira peitoral é evidente, mas sem vestígio de raios. A membrana envolve a porção ventral, sem diferenciação da nadadeira anal. O trato digestivo é evidente com ânus aberto.

A larva com 7,7 mm CP (Fig. 1C) encontra-se no estágio de pós-flexão, com raios da nadadeira caudal plenamente formados, aspecto evidenciado a partir de 7,2 $\mathrm{mm}$ CP. As nadadeiras dorsal, anal e peitorais ainda não apresentam raios evidentes. $\mathrm{O}$ trato digestivo está completamente desenvolvido, sem vestígios de vitelo, e a presença de alimento exógeno se evidencia em larvas a partir de 7,8 $\mathrm{mm} \mathrm{CP}$.

Os raios das nadadeiras peitoral, dorsal e anal encontram-se em formação em larva com $8,0 \mathrm{~mm} \mathrm{CP}$ (Fig. 1D). A bexiga natatória e o trato digestivo são evidentes, com intestino apresentando diversas dobras.

Com 10,1 mm CP (Fig. 1E), ainda na fase de pós-flexão, a larva apresenta corpo mais alto, semelhante ao adulto, com raios da nadadeira anal em fase final de formação. A nadadeira dorsal apresenta aumento do número de raios, porém ainda ausentes em sua porção anterior. A nadadeira ventral ainda não é evidente.

\section{Desenvolvimento morfométrico}

A figura 2 apresenta a regressão linear para as medidas: A) distância pré-anal, pré-dorsal e pré-peitoral, B) comprimento da cabeça e altura do corpo e C) diâmetro do olho, cujos coeficientes de correlação linear de Pearson (r) apresentaram valores superiores a $0,93(\mathrm{p}<0,001)$. O comprimento da cabeça aumenta mais acentuadamente do que sua altura com o desenvolvimento larval.

\section{Relações corporais}

As larvas de $H$. temminckii apresentam variação em suas relações corporais durante o desenvolvimento, sobretudo para a altura do corpo e ponto de inserção das nadadeiras (Fig. 3A). A distância pré-dorsal variou entre 30,8 e $51,2 \%$, a pré-peitoral entre 12,9 e $37,9 \%$ e a pré-anal entre 38,2 e $58,3 \%$ em relação ao comprimento padrão. Estas relações tendem a modificar-se em larvas com CP superior a $10 \mathrm{~mm}$, tendo em vista ainda não terem atingido seu completo desenvolvimento com esse tamanho.

A altura do corpo variou entre 17,2 e $30,4 \%$ em relação ao comprimento padrão, sendo classificada na categoria "moderada" (Fig. 3B).

O comprimento da cabeça variou entre 13,3 e 39,6\% em relação ao comprimento padrão (Fig. 3C), classificando-a na categoria "moderada" até 7,5 mm CP $(<33 \% \mathrm{CP})$ e passando à categoria "grande" acima deste tamanho $(>33 \% \mathrm{CP})$.

O diâmetro do olho variou entre 30,2 e $63,3 \%$ do comprimento da cabeça (Fig. 3D). Esta proporção corporal tende a estabilizar-se em torno de $35 \% \mathrm{CC}$, em larvas com comprimento padrão a partir de $6,0 \mathrm{~mm}$. enquadrando-se na categoria "grande" (>33\% CC).

\section{DISCUSSÃO}

RICHARDS (1985) ao resumir o nível de conhecimento sobre a identificação de ictioplâncton, com base nos dados disponíveis até 1983, tendo analisado a 

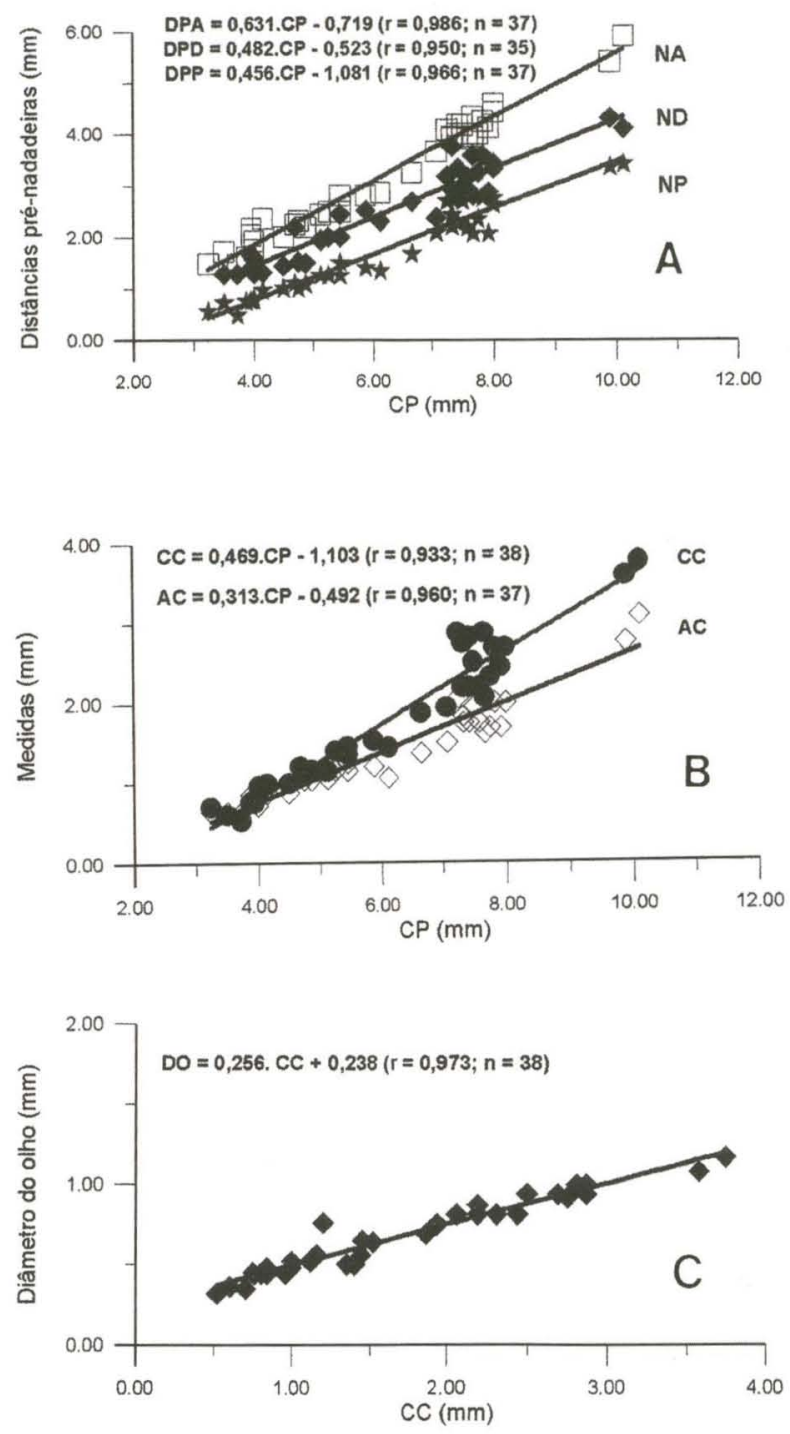

Fig. 2. Regressão linear de: (A) distâncias pré-nadadeiras (NA - anal, ND - dorsal e NP peitoral), (B) comprimento da cabeça (CC) e altura do corpo (AC); (C) diâmetro do olho de Helostoma temminckii.

diferença existente entre os diversos taxa, ressaltou que para algumas espécies e grupos de importância comercial, notadamente marinhos, esse aspecto é bem conhecido. Como exemplo, destacam-se os Clupeiformes de grande importância pesqueira; além de salmonídeos, tunídeos, pleuronectídeos e gadídeos. Esse autor evidenciou, ainda, o pouco conhecimento sobre larvas de peixes de água doce. $\mathrm{Na}$ 
época, apenas 2\% das larvas de Cypriniformes, Characiformes e Siluriformes eram conhecidas, apesar dos dois últimos grupos apresentarem diversas espécies importantes para a pesca e aquicultura. Este conhecimento era restrito a menos de $4 \%$ dos Perciformes, um grupo com diversos taxa de importância pesqueira em todo 0 mundo, sendo que algumas subordens, como a Anabantoidei, não foram sequer contempladas por MOSER et al. (1984), no qual sua análise baseou-se.
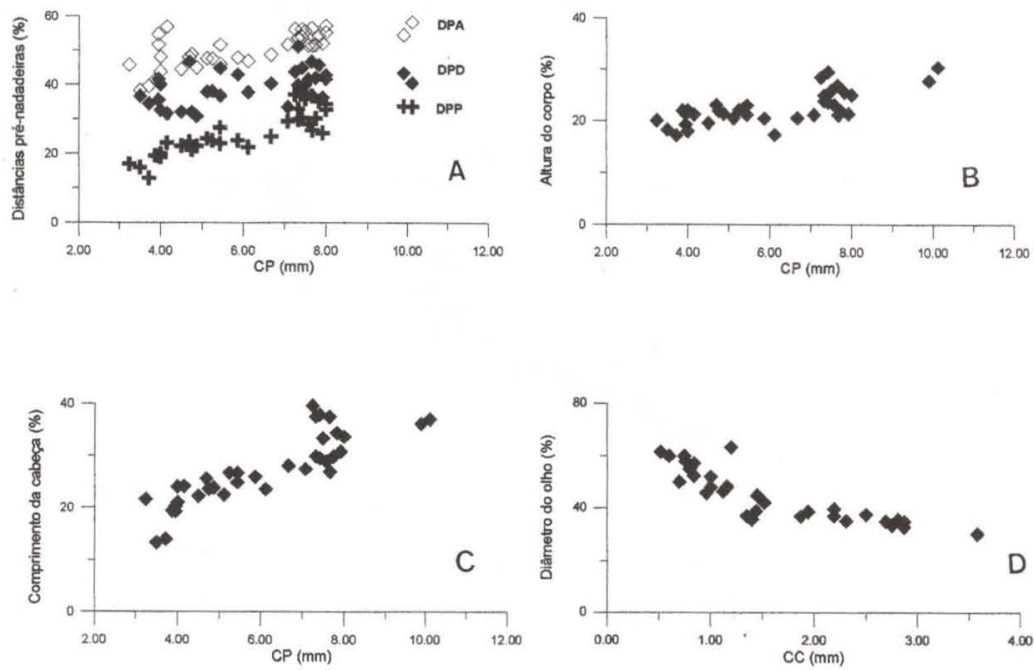

Fig. 3. Relação entre: (A) distâncias pré-nadadeiras (DPD - dorsal, DPA - anal e DPP peitoral); (B) altura do corpo, (C) comprimento da cabeça e o comprimento padrão (CP); (D) diâmetro do olho e o comprimento da cabeça (CC) de Helostoma temminckii.

A identificação de larvas de peixes é uma tarefa difícil e complexa, decorrente da similaridade morfológica entre espécies, da carência de chaves taxonômicas e de literatura especializada, constituindo um processo comparativo por eliminação, onde a obtenção de séries ontogênicas constitui um aspecto fundamental, aspecto este que é favorecido para espécies produzidas em cativeiro, a despeito de possíveis modificações induzidas por fatores ambientais nestas condições (LINDSEY 1988).

Sob o ponto de vista taxonômico, a identificação de larvas de peixes baseia-se em caracteres larvais discerníveis ao nível genérico ou específico, incluindo a forma e o tamanho do corpo nos diferentes estágios de vida, o desenvolvimento de espinhos e ornamentações nos ossos da cabeça e região cleitral, presença de espinhos e raios moles nas nadadeiras, o seu desenvolvimento e o padrão de pigmentação do corpo. O padrão de escamação pode ser relevante, notadamente para espécies com escamas precoces (KENDALL et al. 1984). 
Dados morfométricos e relações corporais têm sido amplamente utilizados na identificação e caracterização de larvas de peixes, aliados a outras características morfológicas e a dados merísticos. A forma geral do corpo das larvas de Perciformes apresenta ampla variação, desde alongada a alta, frequentemente refletindo a forma do corpo do adulto, como o corpo alto em larvas das famílias Chaetodontidae, Pomacanthidae, Menidae, Bramidae e Caristiidae e aquelas alongadas dentre as famílias Congogadidae e Cepolidae. Exceções quanto à semelhança de padrão entre as fases larval e adulta podem ser encontradas, entretanto, entre os Girellidae, Cirrhitidae e Emmelichthyidae (JOHNSON 1984). Dentre os Anabantoidei, as famílias Anabantidae, Helostomatidae, Osphronemidae e Belontidae apresentam espécies com perfil moderado, enquanto Luciocephalidae possui corpo alongado (LAUDER \& LIEM 1983).

O padrão de formação das nadadeiras observado em $H$. temminckii é semelhante ao tipo A apresentado por JOHNSON (1984) para os Percoidei, sendo que o complemento dos raios da dorsal e anal ocorre mais tardiamente, na direção posterior-anterior, seguido do desenvolvimento da peitoral e pélvica. O tamanho na flexão (entre 5,5 e 6,8 $\mathrm{mm} \mathrm{CP}$ ) e o completo desenvolvimento dos raios da caudal é comum à maioria dos Percoidei (JOHNSON 1984).

A presença de espinhos na região cefálica, evidente dentre muitas subordens de Perciformes, representa um caracter útil para a sua diferenciação de larvas de outras ordens de água doce, como Characiformes, Clupeiformes e Siluriformes. Entretanto, dentre os Anabantoidei, a exemplo dos Labroidei, estes parecem ser minimizados ou ausentes (JOHNSON 1984), não tendo sido evidenciados em H. temminckii.

A disponibilidade de ítens alimentares é de extrema importância para a sobrevivência de larvas de peixes durante as fases iniciais da alimentação exógena. Neste sentido, o conhecimento acerca do tamanho no qual ocorre a absorção de vitelo e o início da alimentação exógena pode subsidiar o oferecimento de alimento compatível com o desenvolvimento larval, assim reduzindo sua mortalidade, uma vez satisfeitos outros requerimentos ambientais da espécie.

AGRADECIMENTOS. Agradecemos à Coordenação da Base de Piscicultura do Departamento de Pesca da UFRPE, pelo uso de suas instalações para a realização deste trabalho. Drs. Antonio Lisboa N. Silva (in memoriam) e Rosangela Lessa colaboraram na revisão do manuscrito.

\section{REFERÊNCIAS BIBLIOGRÁFICAS}

Ahlstrom, E.H. \& H.G. Moser. 1976. Eggs and larvae of fishes and their role in systematic investigations and in fisheries. Rev. Trav. Inst. Pêch. Marit. 40 (3): 379-398.

AXELROD, H.R. \& L.P. SCHUI.TZ. 1990. Handbook of tropical aquarium fishes. Neptune City, T.F.H. Pub., $4^{\text {th }}$ ed., $718 \mathrm{p}$.

Blaxter, J.H.S. 1984. Ontogeny, systematics and fisheries. Amer. Soc. Ichthy. Herpet. Spec. Publ. (1): 1-6.

HouDE, E.D. 1987. Fish early life dynamics and recruitment variability. Amer. Fish. Soc. Symposium 2: 17-29.

Johnson, G.D. 1984. Percoidei: development and relationships. Amer. Soc. Ichthy. Herpet. Spec. Pub. (1): 464-498. 
Kendal.t. JR., A.W.; E.H. Ahi.strom \& H.G. Moser. 1984. Early life history stages of fishes and their characters. Amer. Soc. Ichthy. Herpet. Spec. Pub. (1):11-22.

LASKER, R. 1987. Use of fish eggs and larvae in probing some major problems in fisheries and aquaculture. American Fisheries Society Symposium 2: 1-16.

LAuder, G.V. \& K.F. Liem. 1983. The evolution and interrelationships of the Actinopterygian fishes. Bull. Mus. Comp. Zool. 150 (3): 95-197.

Leis, J.M. \& T. TRNSKI. 1989. The larvae of Indo-Pacific shorefishes. Honolulu, Univ. Hawaii Press, $371 \mathrm{p}$.

LindSEY, C.C. 1988. Factors controlling meristic variation, p. 197-274. In: J.D. RANDALL (Ed.). Fish physiology. New York, Academic Press, Vol. XIB.

Moser, H.G.; W.J. Richards; D.M. Cohen; M.P. Fahay; A.W. Kendall Jr. \& S.L. Richardson (Eds). 1984. Ontogeny and systematics of fishes. Amer. Soc. Ichthy. Herpet. Spec. Publ. (1): 1-760.

NELSON, J.S. 1994. Fishes of the world. New York, John Wiley and Sons, $3^{\text {rd }}$ ed., 600p.

Richards, W.J. 1985. Status of the identification of the early life stages of fishes. Bull. Mar. Sci. 37 (2): 756-760.

Recebido em 29.I.1999; aceito em 22.VIII.2000. 